\title{
Investigation into Structural Changes of the Copper Binding Site in Lysyl Oxidase upon Substrate and Inhibitor Docking
}

\author{
M. Lynch and F. Ryvkin \\ Department of Chemistry and Physics, Emmanuel College, 400 Fenway, Boston, MA 02115, USA \\ Correspondence should be addressed to F. Ryvkin; ryvkin@emmanuel.edu
}

Received 28 May 2013; Accepted 19 June 2013

Academic Editors: F. G. Doro, C. L. Liu, and Z. Xiao

Copyright ( 2013 M. Lynch and F. Ryvkin. This is an open access article distributed under the Creative Commons Attribution License, which permits unrestricted use, distribution, and reproduction in any medium, provided the original work is properly cited.

The present paper reports a computational investigation of potential communication between the lysine tyrosylquinone (LTQ) and copper cofactors within lysyl oxidase (LOX). Various substrates and inhibitors of LOX were docked into the active site in our computer generated model of the enzyme. Conformational changes in the vicinity of the copper site as well as changes in the electrostatic environment were identified. The appearance of a canal-like structure involving tyrosine 35 (TYR35) and glutamine 104 (GLN104) residues was shown to be consistent upon docking of a variety of different compounds. Interactions between LOX and its natural substrate, collagen, were also explored through molecular dynamic simulations. The possibility of communication between the organic and inorganic cofactors in LOX was proposed, aiding the ongoing debate regarding the role of copper in the catalytic mechanism of this important enzyme.

\section{Introduction}

Lysyl oxidase (LOX) is a copper containing amine oxidase (EC 1.4.3.13) that catalyzes the oxidation of lysine resulting in the formation of peptidyl $\alpha$-aminoadipic- $\delta$-semialdehyde. Once formed, this product can spontaneously condense to form intra- and inter-chain cross-links in connective tissue matrices $[1,2]$. LOX is synthesized as a $50 \mathrm{kDa}$ proenzyme, secreted into the extracellular environment, and then processed by proteolytic cleavage, resulting in a functional $32 \mathrm{kDa}$ enzyme [3]. Within the LOX structure, two cofactors are of particular interest: a lysine tyrosylquinone (LTQ) and a bound $\mathrm{Cu}(\mathrm{II})$ ion. The copper site has been partially characterized and copper was shown to be coordinated with three specific histidines [4], while LTQ was shown to be formed in a self-processing, copper catalyzed reaction between specific sequential tyrosine and lysine residues in the presence of molecular oxygen [5-7]. Recent research shows developing evidence that the biological role of LOX may extend far beyond that of the structural maturation of elastin and collagen [8-12], demonstrating connections between LOX activity and cancer cell mobility and tumorgenesis [13$15]$.
Many aspects of LOX function are the subject of continuing investigations. Although it has been shown that $\mathrm{Cu}(\mathrm{II})$ is essential for the posttranslational formation of the LTQ cofactor during protein folding prior to proteolytic cleavage [5], whether or not it is imperative to the enzymes functionality has been debated. The first rational, supported by Gacheru et al. [16], presented evidence of copper playing a catalytic role in LOX. It was also reported there that upon copper removal from LOX, the resulting inactive apoenzyme was fully reactivated by reconstitution with $\mathrm{Cu}$ (II), but not by other divalent metal ions. The copper coordination complex in LOX was also postulated using evolutionary arguments in [17], and EPR parameters for $\mathrm{Cu}(\mathrm{II})$ ion in LOX were reported in $[16,18]$ to be similar to that confirmed by Xray crystallography for other copper amine oxidases [19]. A second rational proposed by C. Tang and Klinman [20] suggests that the $\mathrm{Cu}(\mathrm{II})$ ion plays only a structural role within LOX, though both groups attempted similar procedures for copper chelation and assessment of apoenzyme activity. The findings of Tang and Klinman indicate that $\mathrm{Cu}(\mathrm{II})$ is required initially for the formation of LTQ and plays only a structural role in the enzyme afterwards. Even though both theories are supported by experimental data, the possibility that copper 
plays a bifunctional role in LOX, being necessary for both structural stability and catalytic function, is an avenue that cannot be ignored either. Recent methods to obtain pure and active LOX include purification from various sources $[1,2]$, as well as the attempts of its expression using bacterial and insect cells [21-23]. Although these studies have addressed many important characteristics of LOX, their results differ notably from each other [16, 20-23], and the catalytic ability of native and apoenzyme towards elastin and collagen has not yet been explored. The persistent lack of experimental data regarding the three-dimensional (3D) structure of LOX remains a major obstacle to overcome in order to learn more about this important enzyme. At this stage of investigation, the advantages offered by modern development of molecular modeling become an attractive avenue to explore. Based on our computer generated and experimentally validated 3D model of LOX [24], we investigate the activity occurring in LOX following small molecule docking and native substrate molecular dynamics (MD) simulations. We report here the conformational changes that were observed at the $\mathrm{Cu}$ (II) site in LOX and reveal possible communication between both cofactors during catalysis and/or inhibition.

\section{Materials and Methods}

All simulations described in this paper were performed using the Schrödinger Molecular Modeling Software Package [2533]. Energy minimizations were carried out using MacroModel with the OPLS2005 force field, a preconditioned regularized conjugate gradient (PRCG) minimization method, a convergence energy gradient of $0.001 \mathrm{kcal} \mathrm{mol}^{-1}$, and $12 \AA$ nonbonding cutoff limits and were followed by implicit solvation using Impact Soak.

2.1. Model Selection and Preparation. Previously computer generated 3D model of human LOX [24] was used for all simulations without any further modification. The compounds used in docking simulations were selected from the published literature data [34-39], with ligands having substrate and/or inhibitor capabilities towards LOX. All compounds selected were shown to bind directly to LTQ, as substrates or irreversible inhibitors of LOX. The minimized structures were prepared using LigPrep, $\mathrm{pH} 7.8$ to mimic the enzymes optimal catalysis and/or inhibition conditions. Chiralities for the generated ligand were kept as created; the top ligand from the output file was used in docking simulations. Collagen fibril X-ray structures for all molecular dynamics (MD) runs were downloaded from the Protein Databank (PDB), where both a lysine-free $14 \mathrm{kDa}$ collagen fibril (pdb Id: 3b0s) [40] and an $8.5 \mathrm{kDa}$ lysine-containing collagen fiber (pdb Id: $2 \mathrm{klw}$ ) [41] were used.

2.2. Docking Simulations. The receptor docking grid was created through the receptor grid generation program GLIDE and consisted of $15 \AA \times 15 \AA \times 15 \AA$ volume cube grid centered around LTQ. Upon proper grid generation, compounds were docked flexibly through GLIDE ligand docking software, where both nitrogen inversions and ring confirmations were sampled, while nonplanar amide confirmations were penalized. The top pose with the lowest computed GLIDE score was subjected to final structural analysis.

2.3. Molecular Dynamics Simulations. All MD simulations were carried out using Impact dynamics, where the Verlet integration algorithm was implemented. Water molecules remained nonrigid and all bonds were unconstrained throughout the duration of each simulation, where the sample velocity for each trajectory was recorded concurrently every five frames. For efficient screening, 200,000 iterations were permitted, with a time step of $0.001 \mathrm{fs}$ and equilibration time step of $0.010 \mathrm{fs}$ with total simulation time of $20 \mathrm{ps}$, at $300 \mathrm{~K}$. For large substrate MD simulations, variations in residual position were measured between the initial and final frame for each trajectory.

\section{Results and Discussion}

It is a well-established fact [42] that the catalytic processing of peptidyl amine substrates by LOX consists of a two-step, ping-pong mechanism. To do so, the primary amine group on the peptidyl lysine substrate initiates the reaction by reacting with carbonyl oxygen of the LTQ cofactor. Due to the incorporation of this primary amine functional group into many synthetic small molecule substrates and inhibitors, it can be speculated that these compounds behave similar to substrates as they bind to LTQ. In silico modeling, as presented, provides an avenue to explore the interactions occurring between LOX and its substrates and inhibitors. Complete structural characteristics such as the tertiary conformation of LOX, both native and upon small-molecule binding, as well as the quaternary structure of the LOX complex with its macromolecule substrates are still not known. Though some information about the $\mathrm{Cu}$ (II) complex in LOX has been reported $[17,18,24]$, the $\mathrm{Cu}$ (II) site in LOX is only partially characterized. A list of compounds known to be substrates and/or inhibitors to LOX was selected for simulation.

Docking of each compound, shown in Table 1, at the LTQ active site in LOX revealed variations at the molecular surface and in charge distribution. Some of these variations in residue position could be a result of the motion in the LOX random coil structural motifs during the span of each simulation. However, the most significant motion observed in a large majority of simulations could be attributed to two residues. Specifically, the reorientation of glutamine 104 (GLN104) and tyrosine 35 (TYR35) in close proximity to the $\mathrm{Cu}$ (II) site was observed to occur, as a result closing a preexistent canal-like structure identified by a yellow arrow in Figures 1 and 2. Prior to molecular docking at the LTQ site, TYR35 and GLN104 are in an orientation that permitted this canal formation to remain in its open position. After docking and subsequent energy minimization, these two residues shift themselves in a fashion that effectively closes this canal, partitioning the $\mathrm{Cu}$ (II) and LTQ site from one another. The superimposition of two structures before and after small-molecule docking shown in Figure 1 reveals the motion of GLN104 and TYR35 
TABLE 1: The list of various docked substrates and inhibitors, and their effect on the conformational rearrangements GLN104 and TYR35. Distances were measured between the hydroxyl oxygen of TYR35 and the oxygen bound to the $\delta$-carbon of GLN104.

\begin{tabular}{lcc}
\hline Name & Abbreviation & GLN104-TYR35 distance $(\AA)$ \\
\hline Lysyl oxidase (control) & LOX & 9.00 \\
$\beta$-Aminopropionitrile & BAPN & 8.90 \\
Bromoethylamine & BEA & 9.10 \\
Chloroethylamine & CEA & 5.60 \\
Ethylenediamine & EDA & 5.70 \\
3-Aminodihydrofuran-2-one & ADF & 5.70 \\
3-Aminodihydrothiophen-2-one & ADT & 5.90 \\
Nitroethylamine & NEA & 5.50 \\
para-Bromobenzylamine & pBBA & 5.70 \\
para-Chlorobenzylamine & pCBA & 5.50 \\
para-Fluorobenzylamine & pFBA & 5.70 \\
para-Methylbenzylamine & pMBA & 5.50 \\
trans-2-Phenylcyclopropylamine & tPCPA & 5.40 \\
\hline
\end{tabular}

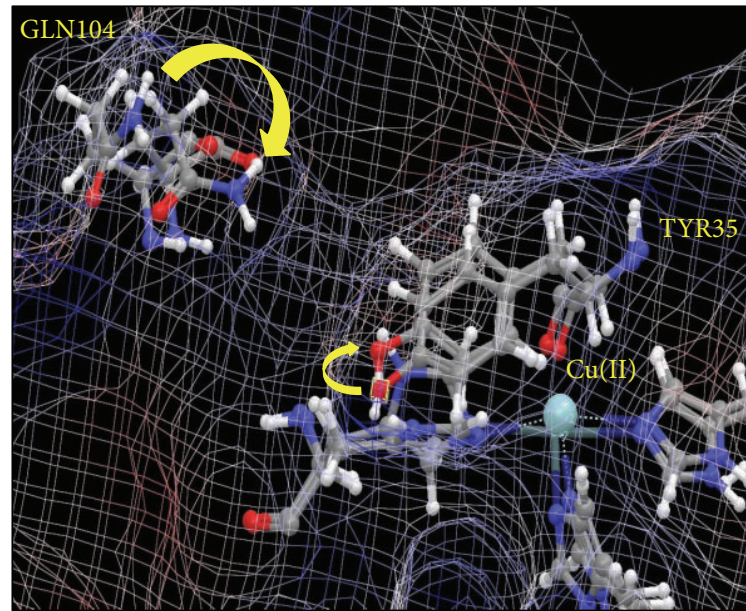

FIGURE 1: Electrostatic surface of two superimposed LOX structures (before and after docking) with specific residues GLN104 and TYR35 displayed near the copper (II) site. Curved arrows indicate residue motion, while the straight arrow indicated position of canal.

that is responsible for the closing of this canal-like structure during docking simulations.

To do so, GLN104 reorients its amide functional group, while TYR35 rotates in a fashion that brings the hydroxyl group in TYR35 in close proximity to the nitrogen bound to the $\delta$-carbon of GLN104. This conformational shift results in the bridging of these two residues, effectively closing the canal. Our simulations raised the possibility that this change in residual separation from $9 \AA$ to $5.6 \AA$ is a connection between LTQ and $\mathrm{Cu}(\mathrm{II})$ that occurs during small-molecule processing. Therefore, all presented evidence indicates the existence of potential communication between the $\mathrm{Cu}$ (II) ion and the LTQ through the significant changes in conformation of two key residues, TYR35 and GLN104. Although LOX can catalytically process and/or become inhibited by many different synthetic compounds, comparing these interactions to those between LOX and its larger natural substrates should be tested. To date, as indicated in the literature, no in vitro studies have investigated the ability of LOX to process the peptidyl lysine present in its naturally occurring substrates collagen and elastin. However, attempts have been made to better probe these interactions through the use of synthetic, larger peptide chains as potential substrates of LOX [43]. Such studies have revealed the preferential bias of LOX to readily catalyze peptides with specific peptidyl anionic residue sequence with respect to the catalytic lysine, where even slight translation of this charge can lead to a significant decrease in the rate of catalysis [43]. Therefore, simulations of LOX with its natural substrate collage have been conducted in this investigation to elucidate a better understanding of the interactions that occur during catalysis at the molecular level. Further, based on results from the small molecule docking simulations which suggest communication between LTQ and $\mathrm{Cu}(\mathrm{II})$, would a similar effect be observed between LOX and collagen? Illustrated in Figure 2, MD simulations of LOX, LOX and a lysine-containing collagen fibril, and LOX with a lysine-free collagen fibril show similarities in residual movement observed in the docking simulations previously described.

In Figure 2(a), a similar canal structure composed of GLN104 and TYR35 residues is present, where upon MD simulations with a collagen fibril containing lysine residues (middle, http://www.rcsb.org/), a rearrangement of GLN104 and TYR35 occurs. Conducting identical MD simulations with a lysine-free collagen fibril (right, http://www.rcsb.org/) does show GLN104 and TYR35 reorientation; however, the similarities in these conformational changes are debatable. Figure 3 outlines the details of these changes in residue position, depicting a superimposition of all three simulations shown in Figure 2. From the analysis of these three superimposed structures, it is evident that during the control simulation of LOX without substrate (Figure 3: blue residues, denoted as $\alpha$ ), the displayed residues remain within their typical distance from one another. Upon MD simulations of LOX and lysine-containing fibril (Figure 3: green residues, 


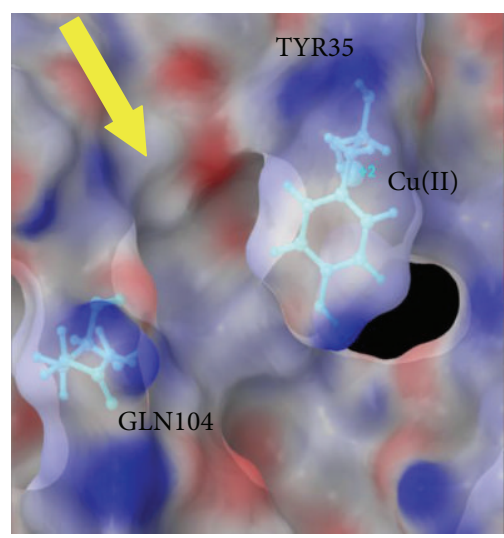

(a)

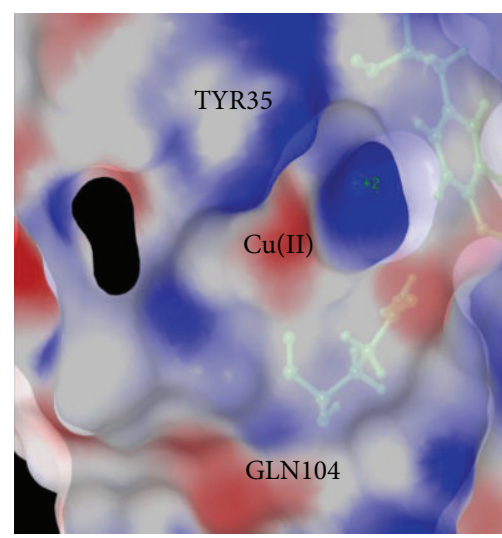

(b)

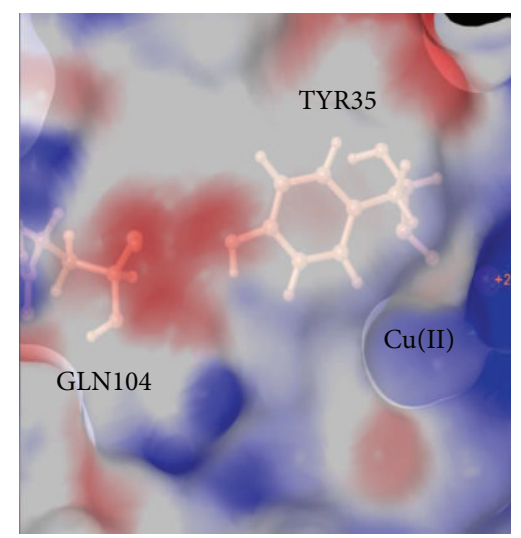

(c)

FIGURE 2: A comparison between the electrostatic surface of LOX upon MD simulations with and without a synthetic collagen fibril. The electrostatic surface of LOX upon MD simulations in the absence of any collagen fibril ((a), the arrow indicated canal position), LOX upon MD simulation with a lysine-containing collagen fibril (b), and LOX upon MD simulations with a lysine-free collagen fibril (c).

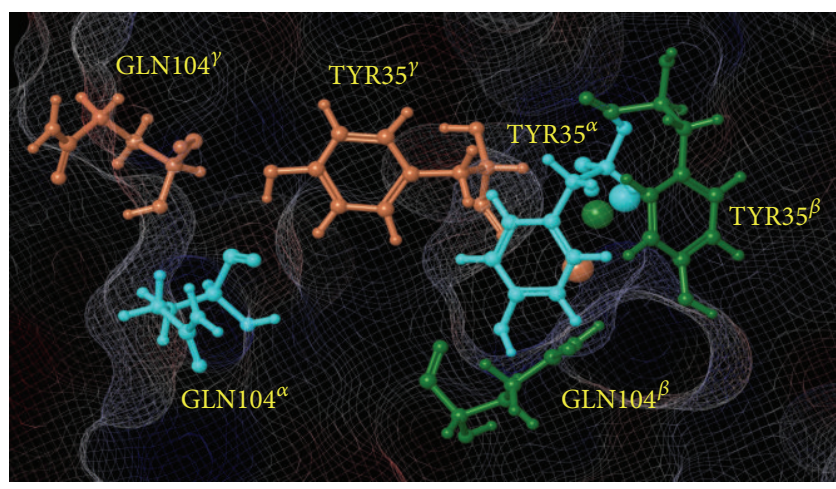

FIGURE 3: The positions of GLN104 and TYR35 residues upon completion of MD simulations. MD of LOX without substrate is colored blue and denoted as $\alpha$, LOX and lysine-containing collagen is colored green and denoted as $\beta$, and LOX and lysine-free collagen is colored red and denoted as $\gamma$.

denoted as $\beta$ ), GLN104 and TYR35 migrate within $4.0 \AA$ from one another and in doing so create a cationic, solvent accessible pocket centered on the $\mathrm{Cu}(\mathrm{II})$ atom. In contrast, MD simulations between LOX and a lysine-free collagen fibril (Figure 3: orange/red residues, denoted as $\gamma$ ) shift left with respect to the $\mathrm{Cu}(\mathrm{II})$ atom in a fashion that creates a more anionic environment in the vicinity of the copper site.

The appearance of a primarily cationic and/or anionic environment in the vicinity of copper site during $\mathrm{MD}$ simulations with the different collagen fibrils identified in Figure 2 may have potential ramifications on the assembly of collagen and elastin within the extracellular matrix. As discussed by Kothapalli and Ramamurthi [44], the structural integrity of the extracellular matrix is heavily dependent on the initial assembly and alignment of tropoelastin fibrils. This alignment of the extracellular proteins promotes proper LOX-catalyzed lysine oxidation, where microfibrils guide the alignment of substrate molecules in a fashion that enables cross-linking and fiber formation to occur. Lysine oxidation is driven predominantly by electrostatic interactions between LOX and substrate [43], suggesting that the appearance of a cationic environment about the copper site as a result of GLN104 and TYR35 rearrangement may be necessary to facilitate proper substrate orientation.

To summarize, this paper presents evidence of the potential communication between $\mathrm{Cu}(\mathrm{II})$ and LTQ during LOX interactions with a primary amine in synthetic small molecules and natural substrates. We hope that further detailed analysis may provide more information about the specific role of copper in this elusive enzyme. It is of particular interest to simulate the enzyme's structural response upon reduction of $\mathrm{Cu}$ (II) to $\mathrm{Cu}(\mathrm{I})$ and how this reduction affects substrate and inhibitor binding. Knowing the enzyme response to reduction, it would be possible to further speculate how GLN104 and TYR35 are involved in the catalysis process of LOX. The work is in progress to further elucidate evidence to support the presence of a possible communication of $\mathrm{Cu}(\mathrm{II})$ with LTQ during the processing of synthetic small molecules and natural substrate collagen.

\section{Conclusion}

The lack of the detailed structural information about the $\mathrm{Cu}$ (II) binding site coordination, its geometry and precise location inside of enzyme precludes the resolution of the debate behind the role of $\mathrm{Cu}$ (II) in primary amine processing by LOX. Although the results presented here do not negate or support either side of this still-standing incongruity, our results reveal that upon docking of amine-containing compounds at the LTQ site, an apparent shift in position of GLN104 and TYR35 residues occurs in the vicinity of the $\mathrm{Cu}$ (II) site. The lysine-containing collagen fibril and LOX complex exhibited similar shifts during MD simulations as observed upon small molecule docking. The presence of a canal-like structure in the proximity of the $\mathrm{Cu}(\mathrm{II})$ site that reorients into a closed position upon perturbing of the LTQ site might suggest possible communication between 
LTQ and $\mathrm{Cu}(\mathrm{II})$ during catalysis. Work is in progress to determine whether this connection between the organic and inorganic cofactors in LOX can be attributed to copper's catalytic, structural, or perhaps multifunctional role within this important enzyme.

\section{Conflict of Interests}

The authors have declared that there is no conflict of interests.

\section{References}

[1] H. M. Kagan, "Characterization and regulation of lysyl oxidase," in Biology of the Extracellular Matrix, R. Mecham, Ed., vol. 1 of Regulation of Matrix Accumulation, pp. 321-398, Academic Press, Orlando, FL, USA, 1986.

[2] H. M. Kagan, K. A. Sullivan, T. A. Olsson III, and A. L. Cronlund, "Purification and properties of four species of lysyl oxidase from bovine aorta," Biochemical Journal, vol. 177, no. 1, pp. 203-214, 1979.

[3] A. D. Cronshaw, L. A. Fothergill-Gilmore, and D. J. S. Hulmes, "The proteolytic processing site of the precursor of lysyl oxidase," Biochemical Journal, vol. 306, no. 1, pp. 279-284, 1995.

[4] K. M. Lopez and F. T. Greenaway, "Identification of the copperbinding ligands of lysyl oxidase," Journal of Neural Transmission, vol. 118, no. 7, pp. 1101-1109, 2011.

[5] J. L. DuBois and J. P. Klinman, "Mechanism of post-translational quinone formation in copper amine oxidases and its relationship to the catalytic turnover," Archives of Biochemistry and Biophysics, vol. 433, no. 1, pp. 255-265, 2005.

[6] J. P. Klinman, "How many ways to craft a cofactor," Proceedings of the National Academy of Sciences of the United States of America, vol. 98, no. 26, pp. 14766-14768, 2001.

[7] J. A. Bollinger, D. E. Brown, and D. M. Dooley, "The formation of lysine tyrosylquinone (LTQ) is a self-processing reaction. Expression and characterization of a Drosophila lysyl oxidase," Biochemistry, vol. 44, no. 35, pp. 11708-11714, 2005.

[8] N. Romero-Chapman, J. Lee, D. Tinker, J. Y. Uriu-Hare, C. L. Keen, and R. R. Rucker, "Purification, properties and influence of dietary copper on accumulation and functional activity of lysyl oxidase in rat skin," Biochemical Journal, vol. 275, no. 3, pp. 657-662, 1991.

[9] G. M. Gilad, H. M. Kagan, and V. H. Gilad, "Evidence for increased lysyl oxidase, the extracellular matrix-forming enzyme, in Alzheimer's disease brain," Neuroscience Letters, vol. 376, no. 3, pp. 210-214, 2005.

[10] H. M. Kagan, "Intra- and extracellular enzymes of collagen biosynthesis as biological and chemical targets in the control of fibrosis," Acta Tropica, vol. 77, no. 1, pp. 147-152, 2000.

[11] Y. Jiang, C. Reynolds, C. Xiao et al., "Dietary copper supplementation reverses hypertrophic cardiomyopathy induced by chronic pressure overload in mice," Journal of Experimental Medicine, vol. 204, no. 3, pp. 657-666, 2007.

[12] H. M. Kagan, "Lysyl oxidase: mechanism, regulation and relationship to liver fibrosis," Pathology Research and Practice, vol. 190, no. 9-10, pp. 910-919, 1994.

[13] Q. Xiao and Ge. Gaoxiang, "Lysyl oxidase, extracellular matrix remodeling and cancer metastasis," Cancer Microenvironment, vol. 5, no. 3, pp. 261-273, 2012.
[14] A. Bondavera, C. M. Downey, F. Ayres et al., "The lysyl oxidase inhibitor, $\beta$-aminopropionitrile, diminishes the metastatic colonization potential of circulating breast cancer cells," Plos One, vol. 4, no. 5, Article ID e5620, 2009.

[15] N. Takeshi, A. Eustace, and C. West, "Lysyl oxidase: from basic science to future cancer treatment," Cell Structure and Function, vol. 37, no. 1, pp. 75-80, 1995.

[16] S. N. Gacheru, P. C. Trackman, M. A. Shah et al., "Structural and catalytic properties of copper of lysyl oxidase," Journal of Biological Chemistry, vol. 265, no. 31, pp. 19022-19026, 1990.

[17] C. J. Krebs and S. A. Krawetz, "Lysyl oxidase copper-talon complex: a model," Biochimica et Biophysica Acta, vol. 1202, no. 1, pp. 7-12, 1993.

[18] F. Ryvkin and F. T. Greenaway, "A peptide model of the copper-binding region of lysyl oxidase," Journal of Inorganic Biochemistry, vol. 98, no. 8, pp. 1427-1435, 2004.

[19] R. Li, J. P. Klinman, and F. S. Mathews, "Copper amine oxidase from Hansenula polymorpha: the crystal structure determined at 2.4 å resolution reveals the active conformation," Structure, vol. 6, no. 3, pp. 293-307, 1998.

[20] C. Tang and J. P. Klinman, "The catalytic function of bovine lysyl oxidase in the absence of copper," Journal of Biological Chemistry, vol. 276, no. 33, pp. 30575-30578, 2001.

[21] S. E. Herwald, F. T. Greenaway, and K. M. Lopez, "Purification of high yields of catalytically active lysyl oxidase directly from Escherichia coli cell culture," Protein Expression and Purification, vol. 74, no. 1, pp. 116-121, 2010.

[22] S. T. Jung, M. S. Kim, J. Y. Seo, H. C. Kim, and Y. Kim, "Purification of enzymatically active human lysyl oxidase and lysyl oxidase-like protein from Escherichia coli inclusion bodies," Protein Expression and Purification, vol. 31, no. 2, pp. 240-246, 2003.

[23] M. S. Kim, S.-S. Kim, S. T. Jung et al., "Expression and purification of enzymatically active forms of the human lysyl oxidase-like protein 4," Journal of Biological Chemistry, vol. 278, no. 52, pp. 52071-52074, 2003.

[24] H. M. Kagan and F. Ryvkin, "Lysyl oxidase and lysyl oxidaselike enzymes," in Biology of the Extracellular Matrix, Springer press, New York, NY, USA, 2011.

[25] Maestro, Version 9. 3, Schrödinger, LLC, New York, NY, USA, 2012.

[26] MacroModel, Version 9. 9, Schrödinger, LLC, New York, NY, USA, 2012.

[27] LigPrep, Version 2. 5, Schrödinger, LLC, New York, NY, USA, 2012.

[28] http://www.rcsb.org/.

[29] Suite 2012: Schrödinger Suite 2011 Protein Preparation Wizard, Epik version 2. 3, Schrödinger, LLC, New York, NY, 2012, Impact version 5. 8, Schrödinger, LLC, New York, NY, USA, 2012, Prime version 3. 1, Schrödinger, LLC, New York, NY, USA, 2012.

[30] R. A. Friesner, R. B. Murphy, M. P. Repasky et al., "Extra precision glide: docking and scoring incorporating a model of hydrophobic enclosure for protein-ligand complexes," Journal of Medicinal Chemistry, vol. 49, no. 21, pp. 6177-6196, 2006.

[31] T. A. Halgren, R. B. Murphy, R. A. Friesner et al., "Glide: a new approach for rapid, accurate docking and scoring. 2. enrichment factors in database screening," Journal of Medicinal Chemistry, vol. 47, no. 7, pp. 1750-1759, 2004.

[32] R. A. Friesner, J. L. Banks, R. B. Murphy et al., "Glide: a new approach for rapid, accurate docking and scoring. 1. Method and assessment of docking accuracy," Journal of Medicinal Chemistry, vol. 47, no. 7, pp. 1739-1749, 2004. 
[33] Suite 2012: Glide, Version 5. 8, Schrödinger, LLC, New York, NY, USA, 2012.

[34] S. N. Gacheru, P. C. Trackman, S. D. Calaman, F. T. Greenaway, and H. M. Kagan, "Vicinal diamines as pyrroloquinoline quinone-directed irreversible inhibitors of lysyl oxidase," Journal of Biological Chemistry, vol. 264, no. 22, pp. 12963-12969, 1989.

[35] G. Liu, K. Nellaiappan, and H. M. Kagan, "Irreversible inhibition of lysyl oxidase by homocysteine thiolactone and its selenium and oxygen analogues: implications for homocystinuria," Journal of Biological Chemistry, vol. 272, no. 51, pp. 32370-32377, 1997.

[36] M. A. Shah, P. C. Trackman, P. M. Gallop, and H. M. Kagan, "Reaction of lysyl oxidase with trans-2-Phenylcyclopropylamine," Journal of Biological Chemistry, vol. 268, no. 16, pp. 1158011585, 1993.

[37] S. Shih Tang, D. E. Simpson, and H. M. Kagan, “ $\beta$-substituted ethylamine derivatives as suicide inhibitors of lysyl oxidase," Journal of Biological Chemistry, vol. 259, no. 2, pp. 975-979, 1984.

[38] S. S. Tang, P. C. Trackman, and H. M. Kagan, "Reaction of aortic lysyl oxidase with $\beta$-aminopropionitrile," Journal of Biological Chemistry, vol. 258, no. 7, pp. 4331-4338, 1983.

[39] P. R. Williamson and H. M. Kagan, "Electronegativity of aromatic amines as a basis for the development of ground state inhibitors of lysyl oxidase," Journal of Biological Chemistry, vol. 262, no. 30, pp. 14520-14524, 1987.

[40] K. K. Okuyama, K. Miyama, K. Mizuno, and H. P. Bachinger, "Crystal structure of (Gly-Pro-Hyp)(9): implications for the collagen molecular model," Biopolymers, vol. 97, no. 8, pp. 607616, 2012.

[41] J. A. Fallas, V. Gauba, and J. D. Hartgerink, "Solution structure of an $\mathrm{ABC}$ collagen heterotrimer reveals a single-register helix stabilized by electrostatic interactions," Journal of Biological Chemistry, vol. 284, no. 39, pp. 26851-26859, 2009.

[42] H. A. Lucero and H. M. Kagan, "Lysyl oxidase: an oxidative enzyme and effector of cell function," Cellular and Molecular Life Sciences, vol. 63, no. 19-20, pp. 2304-2316, 2006.

[43] H. M. Kagan, M. A. Williams, P. R. Williamson, and J. M. Anderson, "Influence of sequence and charge on the specificity of lysyl oxidase toward protein and synthetic peptide substrates," Journal of Biological Chemistry, vol. 259, no. 18, pp. 11203-11207, 1984.

[44] C. R. Kothapalli and A. Ramamurthi, "Copper nanoparticle cues for biomimetic cellular assembly of crosslinked elastin fibers," Acta Biomaterialia, vol. 5, no. 2, pp. 541-553, 2009. 

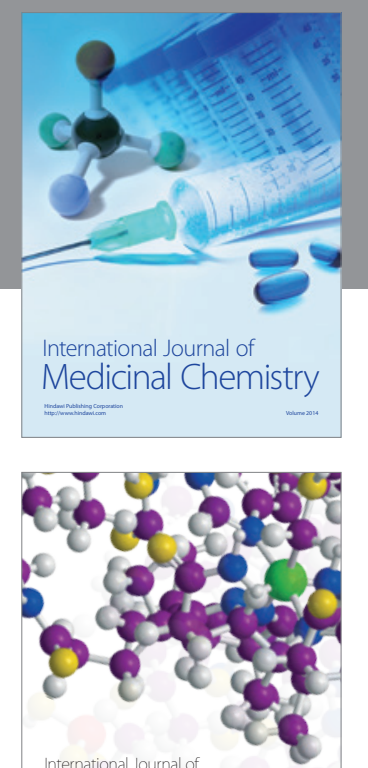

\section{Carbohydrate} Chemistry

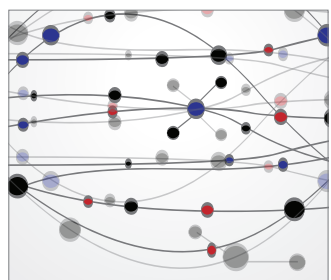

The Scientific World Journal
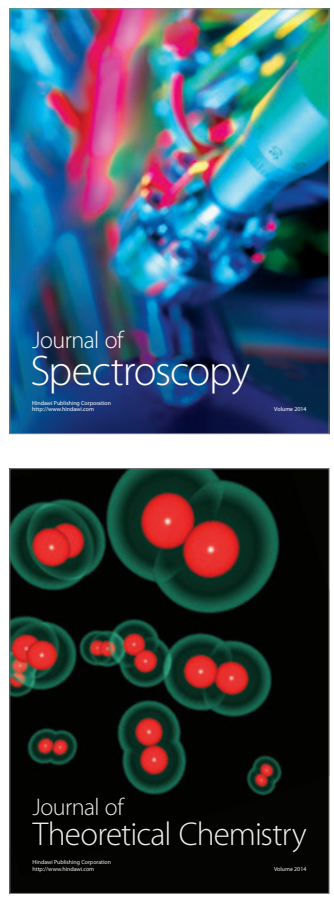
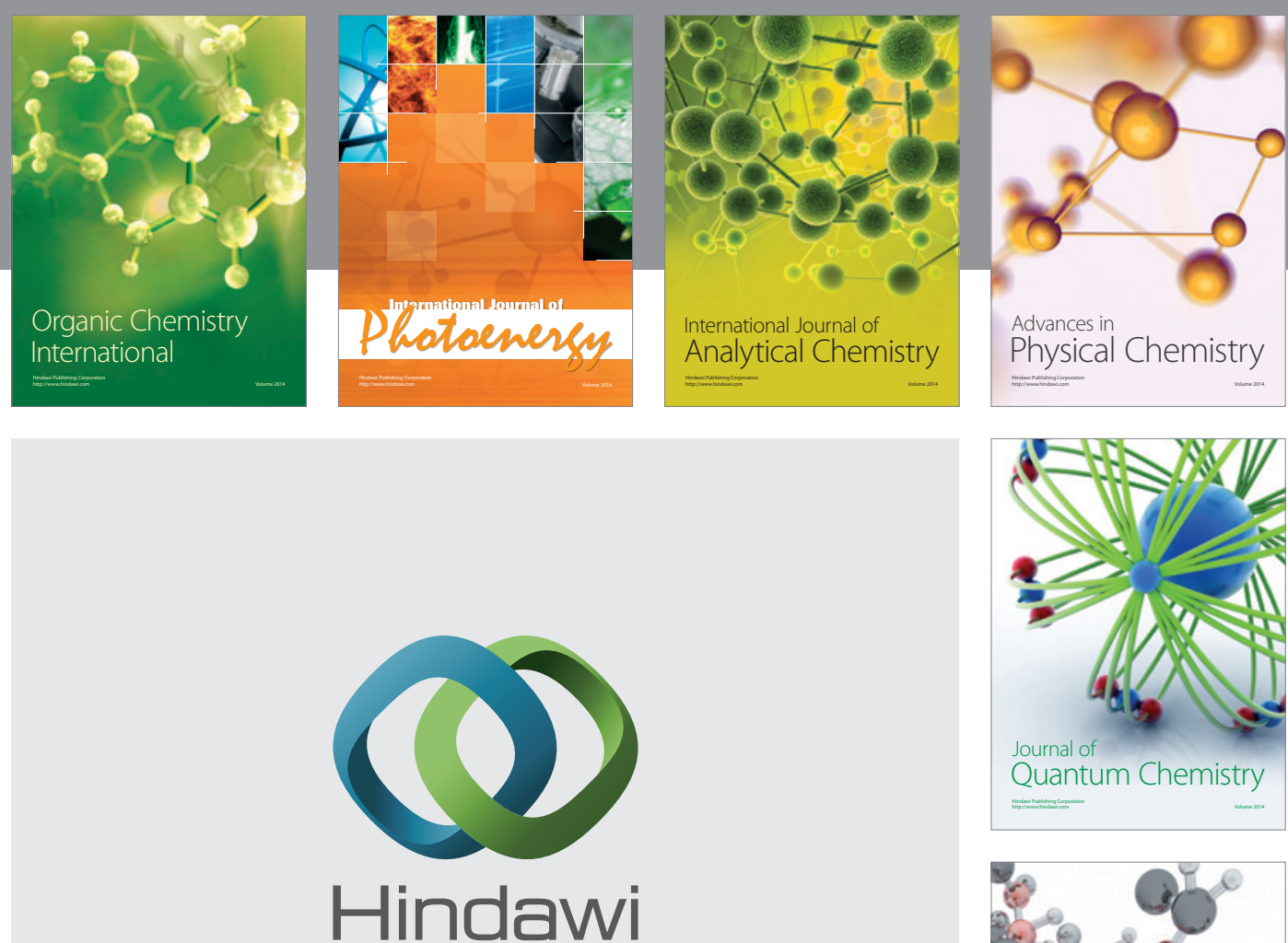

Submit your manuscripts at

http://www.hindawi.com

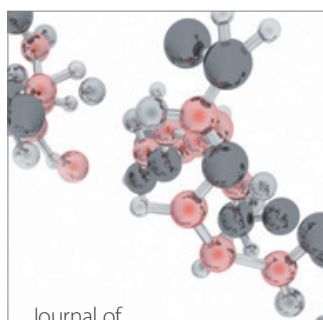

Analytical Methods

in Chemistry

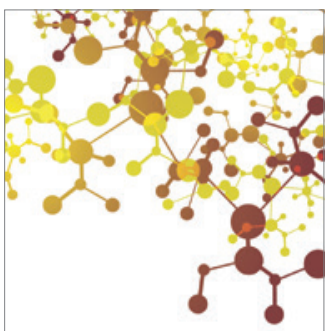

Journal of

Applied Chemistry

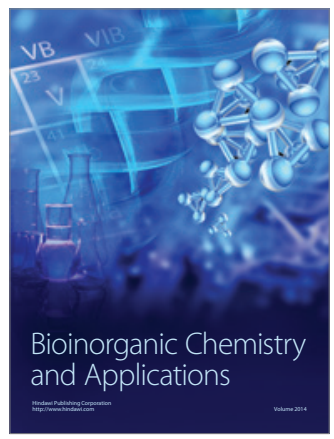

Inorganic Chemistry
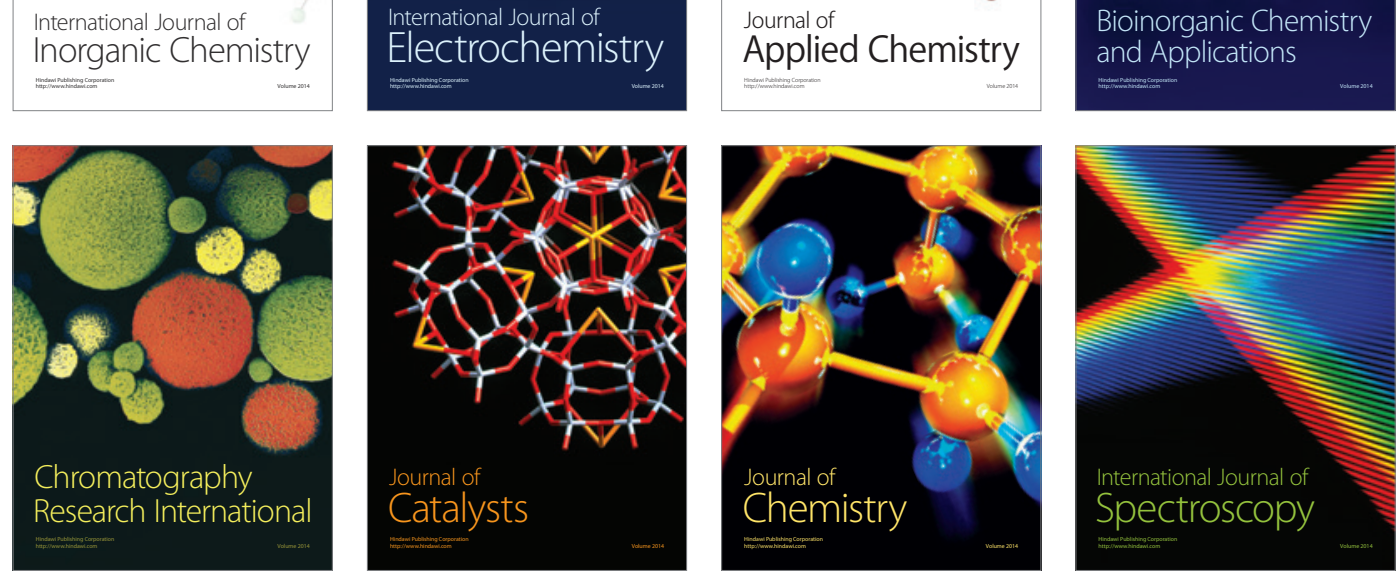